\title{
Motion-capture-like phenomena produced with oppositely drifting gratings
}

\author{
J. TIMOTHY PETERSIK \\ Ripon College, Ripon, Wisconsin
}

\begin{abstract}
Previous research has shown that the apparent motion of a sine-wave grating can be captured by the apparent motion of a second grating with a lower spatial frequency. The present experiments demonstrate that similar capture phenomena can be observed with gratings displaced in real movement. Two gratings that drifted in opposite directions were presented, one of which had constant spatial and temporal frequency and contrast. The subject adjusted the contrast of the second grating until its motion caused the apparent direction of the standard grating to reverse. Four experiments showed that the contrast at which reversal occurred was a minimum when the spatial frequency of the capturing grating was lower than that of the standard and when its temporal frequency was higher than that of the standard. High temporal frequencies of the standard also facilitated capture. It is suggested that capture represents a high-order perceptual strategy.
\end{abstract}

Ramachandran and Anstis (1983) briefly reported the existence of a motion capture phenomenon in which uncorrelated random dots appeared to move coherently with the alternation of phase-shifted low-spatial-frequency gratings. Ramachandran and Cavanagh (1987) made further observations of this phenomenon and confirmed that low spatial frequencies produced more capture than did high frequencies. Furthermore, they discovered that the apparent motion of gratings containing relatively high spatial frequencies could be captured by the apparent motion of gratings with low spatial frequencies, regardless of the direction of displacement of the high-frequency grating.

In the present exploratory experiments, we asked whether motion-capture-like phenomena could be observed between pairs of gratings made to drift in opposite directions. The range of spatial and temporal frequencies used produced a wide variety of dynamic spatial envelopes. We reasoned that if similar capture-like phenomena could be obtained with a number of different spatiotemporal waveforms, even when the inducing stimuli could be expected to stimulate nonoverlapping motion channels in the visual system, then motion capture might reflect a higher order perceptual processing strategy.

\section{GENERAL METHOD}

We started with a standard grating of fixed spatial and temporal frequency and contrast drifting in one direction on an oscilloscope screen. The contrast of a second, oppositely moving, grating was then raised by the subject until (1) the entire display appeared to move in the direction of the variable-contrast (capturing) grating, (2) the initial grating (standard) appeared to stop moving, or (3) the original motion of the standard was not visible without a great deal of attention. The first of these criteria was used by far the most frequently. The second and third criteria were used less often and usually only when the spatial frequencies of the inducing gratings differed a great deal. In Experiment 1, the contrast at which the second, oppositely moving, grating was able to

Correspondence may be sent to J. Timothy Petersik, Department of Psychology, Ripon College, P.O. Box 248, Ripon, WI 54971. capture the motion of the standard grating was measured as a function of the spatial and temporal frequency of the oppositely moving grating. Experiments 2 and 3 were conducted to examine motion capture with a different spatial frequency of the standard stimulus and to control fixation (Experiment 3). Experiment 4 was conducted to further explore the influence of temporal frequency on the motion-capture effect.

\section{Subjects}

Three trained psychophysical observers, including the author, participated in each experiment. Each subject had normal or corrected-tonormal vision.

\section{Stimuli and Apparatus}

Sine-wave gratings were produced in a conventional manner by a microcomputer-based system (originally described by Fritsch \& Keck, 1978) and were displayed on a Hewlett-Packard Model 1340-A oscilloscope, coated with a rapid-decay (P-31) phosphor under control of an SYM-1 (Synertek Systems) microcomputer. The space average luminance of all displays was $20 \mathrm{~cd} / \mathrm{m}^{2}$.

Each trial consisted of the presentation of a rightward-drifting standard grating of a given spatial and temporal frequency (varied across experiments). Its Michelson contrast was constant at $30 \%$. The contrast of a second, oppositely moving (capturing), grating was under the control of the subject. Its spatial and temporal frequencies varied across experiments.

The observer sat in a small booth and looked through two eyepieces at the oscilloscope screen, a distance of $79 \mathrm{~cm}$ away. A small tunnel between the oscilloscope and eyepieces ensured that only the screen was visible. The screen subtended $7^{\circ} \times 9^{\circ}$ arc.

\section{Procedure}

Each trial began with the rightward motion of the standard stimulus. After a few moments of viewing, the observer gradually raised the contrast of the oppositely drifting capturing grating until capture was observed. The reversal contrast was subsequently recorded. Each observer had previously been given a minimum of $5 \mathrm{~h}$ of practice at this task to aid the control of fixation. To avoid motion aftereffects, between trials, the subjects stared for $2 \mathrm{~min}$ at a stationary grating of the same spatial frequency as the standard. Each data point reported below is based on 60 trials, 20 per observer.

\section{RESULTS}

\section{Experiment 1}

The spatial and temporal frequency of the standard grating were .44 cycles/degree (cpd) and $5 \mathrm{~Hz}$, respectively. 
The spatial and temporal frequency of the capturing grating varied randomly from .11 to $1.78 \mathrm{cpd}$ and 2 to $10 \mathrm{~Hz}$, respectively. Individual standard deviations were generally low (2\%-7\%) for spatial frequencies at and below that of the standard stimulus. For the higher frequencies, standard deviations ranged from $6 \%$ to $19 \%$, which conformed to subjective impressions that motion capture was easier to observe with low-spatial-frequency capturing gratings. Figure 1 presents mean reversal contrast (lowest contrast at which capture occurred) as a function of the spatial frequency of the opposite-motion capturing grating. As can be seen, reversal contrast was lowest when the spatial frequency of the capturing grating matched that of the standard grating and increased as spatial frequency deviated from the standard. For spatial frequencies lower than the standard, reversal contrast was inversely related to temporal frequency. For spatial frequencies higher than the standard, there was little effect of temporal frequency.

These results suggest that motion capture can be obtained with gratings that are both lower and higher in spatial frequency than the standard. They also suggest that, at least for the low-spatial-frequency capturing gratings, motion capture is obtained easier with higher temporal frequencies.

\section{Experiments 2 and 3}

In both experiments, the standard grating had a spatial frequency of $1.78 \mathrm{cpd}$ and drifted with a temporal frequency of $5 \mathrm{~Hz}$. The spatial frequencies of the oppositemotion grating ranged from .22 to $7.12 \mathrm{cpd}$. Its temporal frequency was 2,5 , or $10 \mathrm{~Hz}$. As a control for fixation, in Experiment 3, a solid fixation circle (12 in. in diameter) was positioned on the center of the CRT.

Figure 2 shows the average reversal contrasts of Experiment 2. As in Experiment 1, the temporal frequency of the capturing grating had an effect only below the spatial frequency of the standard. When comparing the results

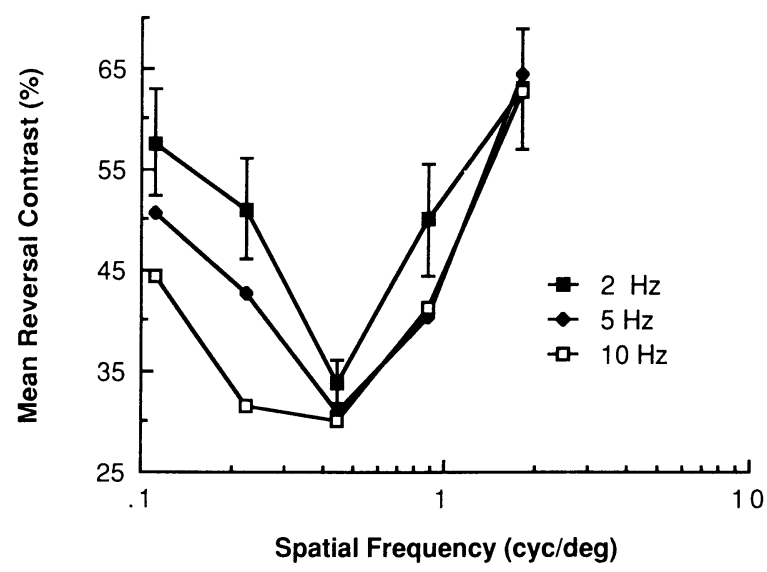

Figure 1. Mean contrast required to produce motion capture as a function of the spatial frequency of the capturing (oppositely moving) grating, Experiment 1. The temporal frequency of the capturing grating is the curve parameter. Error bars represent \pm 1 group standard deviation.

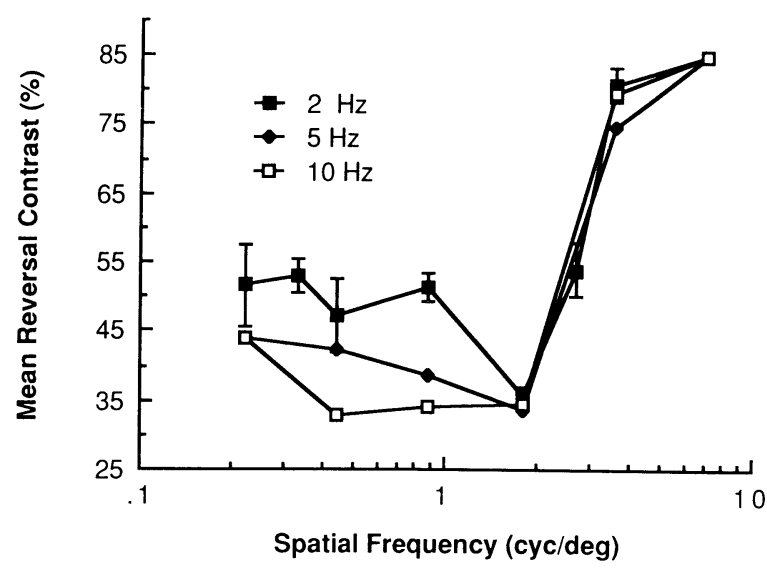

Figure 2. Mean reversal contrast as a function of the spatial frequency of the capturing grating, Experiment 2. The temporal frequency of the capturing grating is the curve parameter. Error bars represent \pm 1 group standard deviation.

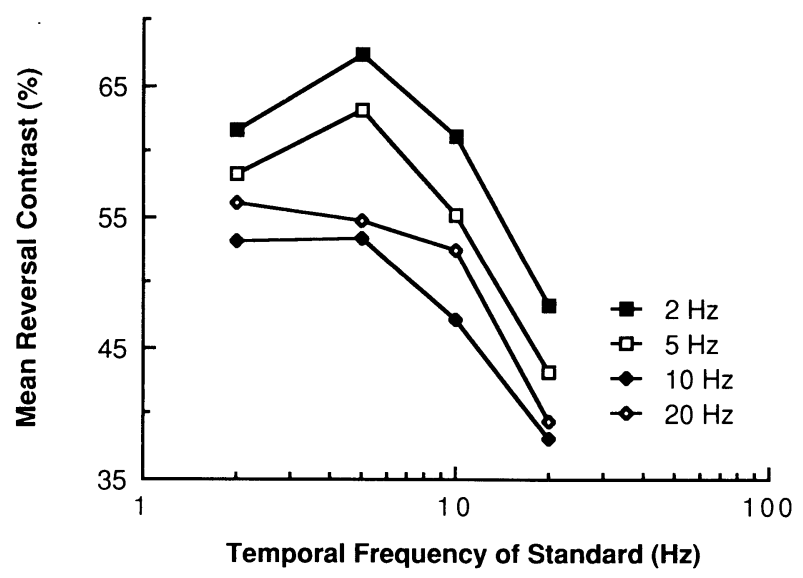

Figure 3. Mean reversal contrast as a function of the temporal frequency of the standard grating, Experiment 3. The temporal frequency of the capturing grating is the curve parameter.

of Experiment 1 with those of Experiment 2, we found that, in Experiment 2, there was a stronger tendency for the slopes of the curves above the standard spatial frequency to be steeper than the slopes of the curves below. This again supports the notion that motion capture is obtained more easily with gratings lower in spatial frequency than that of the standard, captured, grating. These results also suggest that as the spatial frequency of the standard grating is increased, it becomes increasingly more difficult to obtain capture with high-spatial-frequency capturing gratings.

The data of Experiment 3 virtually overlapped those of Experiment 2. Hence, it can be concluded that the motioncapture effects of the first two experiments were likely not to have been influenced by eye movements.

\section{Experiment 4}

The spatial frequency of the standard grating was $1.78 \mathrm{cpd}$. The opposite-motion grating had a spatial fre- 
quency of $1.34 \mathrm{cpd}$. The temporal frequencies of both gratings varied among $2,5,10$, and $20 \mathrm{~Hz}$.

The average reversal contrasts are shown in Figure 3. The figure shows that, in general, increases in the temporal frequency of the capturing grating up to about $10 \mathrm{~Hz}$ result in capture occurring at progressively lower contrasts. Increases in the temporal frequency of the standard grating, especially beyond $5 \mathrm{~Hz}$, also facilitated its capture. The major exception occurred at values of $2 \mathrm{~Hz}$ and $5 \mathrm{~Hz}$ for both gratings. The pattern of results suggests that high temporal frequencies (above $5 \mathrm{~Hz}$ ) of either or both of the oppositely moving gratings facilitate motion capture. For lower temporal frequencies, the data suggest a tuning effect, in which gratings at or near $5 \mathrm{~Hz}$ are resistant to capture.

\section{DISCUSSION}

These experiments show that a motion-capture-like effect can be obtained with grating stimuli in real movement. The influence of the spatial and temporal frequencies of the captured (standard) and capturing gratings can be summarized as follows: (1) Capture requires somewhat less contrast when the spatial frequency of the capturing grating is lower than that of the standard, and this effect is enhanced by higher temporal frequencies; (2) gratings of a spatial frequency higher than that of the standard are able to produce capture, although they require more contrast; here, temporal frequency has little or no effect; (3) for two oppositely moving gratings of similar spatial frequency and a low temporal frequency of the capturing grating, the contrast required to produce capture is an inverted-U-shaped function of the temporal frequency of the standard; and (4) when the two gratings have a high spatial frequency and the capturing grating has a high temporal frequency, capture is facilitated by high temporal frequencies of the standard.

The present capture phenomenon is similar to one reported by Adelson and Movshon (1982), in which gratings of different orientations com- bined to produce a coherently moving plaid grating. Hildreth (1984) suggested that such coherence occurs when analysis of the two moving gratings occurs within the same broadly tuned spatial-frequency channel(s). Such an analysis could equally well apply to the present results. Indeed, when the spatial frequencies of the inducing gratings differed much more than the extremes reported here, the two gratings were always seen to move independently, producing a perceptual transparency. However, capture seemed to be a relatively ubiquitous phenomenon with these above-threshold stimuli, and it was produced with a wide variety of spatiotemporal envelopes, which suggests that motion capture may represent a higher order processing strategy. Such a strategy would signal a dominant stream of motion to an observer in an otherwise ambiguous dynamic image. It is consistent with the hypothesis that high temporal frequencies facilitate motion capture, especially if it is assumed that motion-detecting mechanisms become increasingly dominant over pattern detecting mechanisms as temporal frequency is raised.

Given this hypothesis, the next step in research of this type should be to determine the tuning functions of psychophysical channels stimulated by the component gratings in a variety of motion-capture displays. Evidence that capture is obtained when nonoverlapping channels are stimulated, particularly when the direction of capture is not predicted by the spatiotemporal waveform of the composite stimulus, would support the above hypothesis.

\section{REFERENCES}

Adelson, E. H., \& Movshon, J. A. (1982). Phenomenal coherence of moving patterns. Nature, 300, 523-525.

FriTsCH, K., \& KeCK, M. (1978). Grating generation by microcomputer. Vision Research, 18, 1083-1085.

HiLdRETH, E. C. (1984). The measurement of visual motion. Cambridge, MA: MIT Press.

Ramachandran, V. S., \& ANstis, S. M. (1983). Displacement thresholds for coherent apparent motion in random-dot patterns. Vision Research, 23, 1719-1724.

Ramachandran, V. S., \& Cavanagh, P. (1987). Motion capture anisotropy. Vision Research, 27, 97-106.

(Manuscript received January 26, 1989.) 